\title{
Sprachspiele von Paul Maar im Unterricht. Ein Unterrichtsvorschlag
}

\author{
Jana Baroková
}

This article deals with the German lyrics for children. At first, various forms of the lyrics, especially nursery rhyme at school and by the own lecture are treated. Based on the book Jaguar und Neinguar by Paul Maar, various forms of the lyrics for children are categorized. Concrete forms like spoonerism, counting-out rhyme, tongue twister, puzzles etc. are methodologically and didactically drawn up for the lessons.

German lyrics for children - nursery rhymes - Paul Maar-Jaguar und Neinguar - spoonerism counting-out rhyme - tongue twister - puzzle - teaching techniques and didactics

Dieser Artikel befasst sich mit der deutschen Kinderlyrik. Zuerst wird die Rolle von diversen Formen des Kinderreims in der Schule und bei der eigenen Lektüre behandelt. Anhand der Gedichtsammlung Jaguar und Neinguar befasse ich mich mit den Sprachspielen von Paul Maar. Zuerst werden diverse Formen der Kinderlyrik kategorisiert. Konkrete Formen der Sprachspiele wie Schüttelreim, Abzählreim, Zungenbrecher, Rätsel etc. werden für den Unterricht methodisch-didaktisch vorgeschlagen.

Deutsche Kinderlyrik - Kinderreim - Paul Maar - Jaguar und Neinguar - Schüttelreim Abzählreim - Zungenbrecher - Rätsel - methodisch-didaktische Vorschläge

\section{Bedeutung und Rolle der Lyrik im Leben der Kinder}

Lyrik in der Kindheit - das heißt sicher nicht nur das Auswendiglernen und Vortragen von Gedichten in der Schule, sondern primär Freude am gesprochenen Wort, am Spiel mit Worten und ungewöhnlichen Wortverbindungen und -kombinationen. Durch die Beherrschung der verschiedenen lyrischen Elemente erreicht das Kind unter seinen Gleichaltrigen soziale Kompetenz und Anerkennung. Gefördert werden die Sprachmelodie und der Sprechrhythmus. Die semantische Spanne des Begriffs „Kinderlyrik“ reicht vom einfachsten Fingerspiel, über Wiegenlieder, Spiellieder, Kniereiter und Nonsens-Gedichte bis zur intentionalen Ding- und Reflexionslyrik. Manche Texte entfalten ihre Wirkung erst in visueller Form - als konkrete bzw. experimentelle Poesie. Im vorliegenden Artikel möchte ich zeigen, wie die Kinderlyrik in der Schule angewendet werden kann. Zu diesem Zweck habe ich die Gedichte des deutschen Autors Paul Maar ausgewählt.

\section{Sprachspiele im Unterricht}

Die Lektüre von Originaltexten in der Fremdsprache erweitert die Lesekompetenz und bereichert die Persönlichkeit des Lesers. Das gilt in noch höherem Maße für die Sprachspiele, die für die Entwicklung des Kindes äußerst förderlich sind. 
Sprachspiele sind Spiele mit Lauten, Buchstaben, Wörtern, Sätzen, ungewöhnlichen Wortkombinationen und Satzzusammenhängen, die zum Komischen, zum Phantastischen und zur Verfremdung führen. Monika BORN (1996) unterscheidet das Sprachspiel als Ergebnis des Spielens mit Sprache und Spielen mit der Sprache als den Prozess selbst, d. i. den kreativen Umgang mit Sprachspielen. Beides hängt jedoch eng zusammen, da Sprachspiele in gelesenen Texten die Leser wiederum selbst zum eigenen Spiel mit der Sprache anregen. Diverse Formen von einfachster Lyrik - Lautgedichte, Zungenbrecher, Kniereiter, Lügengedichte, Abzählverse, Schüttelreime - beinhalten sprachspielerische Elemente.

Aus der Sicht des Kindes besteht der Sinn des Spielens mit der Sprache vor allem darin, dass mit den Lauten manipuliert wird: Es entsteht „schöner Unsinn“, das Kind freut sich über die spielerischen Abweichungen von der Norm. Durch das Spielen mit Worten entsteht gereimte und rhythmisierte Sprache, Wortneubildungen und -verdrehungen, semantische Verkehrungen. Wörter werden von hinten nach vorn gelesen, die Sprechweise der Erwachsenen wird nachgeahmt, was viel Spaß mit sich bringt.

Eltern und Pädagogen schätzen am Spielen mit Sprache vor allem den kreativen Umgang, wodurch primär die Freude an sprachlicher Kreativität und an Literatur gefördert wird. Kinder freuen sich an sprachlichem Witz und komischen Wortverdrehungen, wenn sie Wörter von hinten nach vorn lesen können und wenn sie die Sprache der Erwachsenen imitieren können. Im Prozess des Literaturunterrichts trägt die Erfassung von Texten zu der Fähigkeit bei, die Absicht des Autors und den Aufbau des Textes besser zu begreifen, und weckt die Bereitschaft, sich mit den Texten kritisch auseinanderzusetzen. Letztendlich verbessert diese Fertigkeit die Lesetechnik und Rechtschreibung.

Obwohl Lernziele sehr wichtig sind, darf meiner Ansicht nach nicht vergessen werden, dass es beim Sprachspielen primär um das Vergnügen am eigentlichen sprachspielerischen Prozess geht, um die Freude an dieser Betätigung. Beim methodischen Umgang mit Sprachspielen sollte daher berücksichtigt werden, dass Sprachspiele als Alternative und Ergänzung zum zielgerichteten Lernen dienen sollten, wo kein Zwang herrscht, sondern das Kind entsprechend der angebotenen Modelle seine eigenen Versuche und Kreationen erproben kann. Der Lehrer kann dabei an verschiedenen Stellen korrigierend eingreifen.

In den folgenden Ausführungen werde ich mich der Kinderlyrik von Paul Maar widmen, in der Sprachspielen, Kinderreimen und Nonsens-Gedichten ein wichtiger Stellenwert zukommt. Zunächst aber ein paar Worte über Paul Maar selbst.

\section{Paul Maar}

Paul Maar wurde 1937 in Schweinfurt geboren. Als Kind erlebte er den Tod der Mutter, den Krieg und die Not in der Nachkriegszeit. Nach dem Abitur studierte 
er Kunstgeschichte und Malerei. 1968 veröffentlichte er sein erstes Kinderbuch Der tätowierte Hund und gleichzeitig begann er Kindertheaterstücke und Drehbücher für Kindersendungen im Fernsehen zu schreiben. Der Erfolg des 1973 erschienenen Kinderbuches Eine Woche voller Samstage hält bis heute an. Seit 1976 arbeitet Paul Maar als freier Schriftsteller und Illustrator. Er schreibt sowohl realistische Jugendromane, wie z. B. Andere Kinder wohnen auch bei ihren Eltern (1976) oder Kartoffelkäferzeiten (1990), als auch Geschichten mit phantastischen Elementen und einer starken eskapistischen Funktion, wie z. B. Lippels Traum (1984).

Schon als Kunststudent hatte sich Paul Maar der konkreten Poesie zugewandt, wobei er unter anderem von Ernst Jandl beeinflusst wurde.

Sein lyrisches Schaffen ist sehr umfangreich. Auf diesem Gebiet widmet er sich vor allem den Sprachspielen und dem Kinderreim, weil er darin eine große Herausforderung für die junge Generation sieht. In einem Gespräch mit den Kinderbuchautoren Gudrun Mebs und Gunter Preußäußert er sich zur zunehmenden Spracharmut der Kinder von heute wie folgt:

Ich habe eine Statistik gelesen, dass vor zwanzig Jahren $5 \%$ der Kindergartenkinder sprachgestört waren, jetzt sind es danach $26 \%$, und die Tendenz ist steigend. Diese Zunahme hängt wahrscheinlich damit zusammen, dass die Kommunikation im Elternhaus immer weniger wird und man auch die kleinen Kinder vor die ,Teletubbies' setzt. Sie können dann zwar einfache Worte sprechen, weil sie aber immer nur hören und nicht gefordert werden, selbst etwas zu sagen, sind sie nicht mehr fähig, ganze Sätze zu formulieren, haben zum Teil auch Artikulationsschwierigkeiten, sprechen Wörter falsch aus. [...] Ich denke, auf die Sprache zu achten, ist ein Teil unserer Arbeit, den wir nicht vernachlässigen dürfen.

(Paul Maar, zit. nach PAYRHUBER: 2000, 103)

Die im Jahre 2007 veröffentlichte Gedichtsammlung Jaguar und Neinguar enthält diverse Formen des Kinderreims, wie Abzählverse, Rätselgedichte, Bildgedichte, Nonsens, reflexive Poesie, gereimte Reminiszenzen an die wohl bekanntesten „SamsGeschichten" des Autors (vgl. unten) und andere. In seinen Sprachspielen ist neben dem hintergründigen Witz oft eine leise Poesie versteckt. Schon die Gestaltung des Einbandes und des Titels des Buches deutet an, dass es sich um ein Sprachbastelbuch handelt. Paul Maar äußert sich selbst dazu, wie ihm der Titel des Buches eingefallen ist, wie folgt: „Ich denke, wenn es einen JAguar gibt, dann gibt es sicher auch einen NEINguar."

1 So äußerte sich Paul Maar während seiner Lesung zum oben genannten Buch auf der Internationalen Tagung der Deutschlehrer und -lehrerinnen in Jena, 2009, wo auch die Autorin dieses Artikels anwesend war. 
Das Buch ist mit farbigen Illustrationen von Ute Krause (geb. 1960) versehen, die Cartoons zeichnet und Drehbücher schreibt, und deren Werk vielfach ausgezeichnet worden ist.

\section{Komik und Spiel mit Sprache als Merkmale des Schaffens von Paul Maar}

Paul Maar arbeitet in seinen Kindergedichten mit verschiedenen Arten von Komik. Er bereichert die Handlung durch Wendungen und Fügungen, es entsteht eine konkrete Situationskomik. Seine Texte sind voll von überraschenden Ideen, von gespielter Naivität und Witz. Besonders typisch für das Schaffen des Autors sind Reimereien und kleine Gedichte, in denen das Sams - der wohl bekannteste Protagonist seiner Bücher - auftritt. Das Sams gehört zu den ungewöhnlichsten Wesen der phantastischen Kinderliteratur und wird von den Kinder vorbehaltlos mit Liebe angenommen, und dies, obwohl es mit seinem Aussehen zum Außenseiter prädestiniert ist. Der Grund für diese Akzeptanz liegt wohl darin, dass dieses rein ausgedachte Wesen aus einer phantastischen Welt sich über jegliche Normen, Prinzipien und Regeln der Gesellschaft hinwegsetzt und eigene Initiative entwickelt. Es steht im absoluten Gegensatz zu den sich in ihrem Umfeld oft angepasst und unterwürfig verhaltenden Elternfiguren, die auf der anderen Seite ihre Kinder als „Autoritätspersonen“ schikanieren und unterdrücken.

Im Abschnitt Nun kommt das Sams im Buch Jaguar und Neinguar erscheinen Gedichte über das Sams als ein- bis mehrstrophig, die aus drei- oder vierhebigen Jamben, Trochäen oder wechselnden Versfüßen bestehen, mit Paarreimen, Kreuzreimen oder Mehrfachreimen, mal rein, mal assonant, mal unrein. Es wird also eine Fülle lyrischer Möglichkeiten realisiert. (vgl. LANGE: 1996, 145). Der Inhalt der Reime ist ausgesprochen humorvoll, sie drücken sprachspielerische Lust aus, was meiner Meinung nach das folgende Gedicht gut zeigt:

Sams,

und wie alt bist du?

Du fragst nach dem Alter?

Das ist doch ganz klar:

Ich bin ein Jahr älter, als ich letztes Jahr war.

(MAAR: 2007, 65)

Die Figur des Sams zeigt den Kindern, dass die Autorität der Erwachsenen nicht allmächtig ist und dass das Kind die Probleme selbst bewältigen kann. Dadurch gewinnen die Sams-Verse eine deutlich emanzipatorische Funktion. Wünsche, Bedürfnisse und Triebe müssen nicht fortlaufend unterdrückt werden, sondern man darf sie ausleben und stellvertretend befriedigen, wie das nächste Gedicht zeigt: 
Sams,

wie ist das mit dem Wünschen?

Wenn du eigne Wünsche hast, dann greif ruhig nach den Sternen.

Wünsch dir alles, was du willst.

Das kannst du von mir lernen.

Doch wenn du einem Böses wünschst, zum Beispiel deinem Feind, dann wird das leider nicht erfüllt, dann wird der Wunsch verneint.

(MAAR: 2007, 68)

\section{Klassifikation der Gedichte}

In den nächsten Absätzen werde ich versuchen, die Gedichte aus dem Gedichtband Jaguar und Neinguar unter didaktisch-methodischen Aspekten unter die Lupe zu nehmen. Die Texte haben ein breites Anforderungsniveau, so dass Kinder unterschiedlichen Alters angesprochen werden. Weil das Buch für die Klassenarbeit bestimmt ist, sollte es bereits längere Zeit vorher den Schülern in der Klassenbibliothek zur Verfügung stehen, damit sie sich mit den einzelnen Gedichten vertraut machen können.

Arten von Lyrik, die in diesem Buch erscheinen, sind:

- Ding- und Reflexionslyrik im Abschnitt Die gewohnten Dinge (S. 7-24),

- Nonsens-Gedichte und assoziative Kinderlyrik im Abschnitt Aus dem Drachenleben (S. 27-52),

- Schüttelreime im gleichnamigen Abschnitt (S. 55-58),

- Spiel mit Buchstaben, Abzählreime und Reflexionslyrik im Abschnitt Nun kommt das Sams (S. 61-78),

- reflexive Lyrik und Naturlyrik in den Abschnitten Der Abend, die Nacht (S. 81-90) und Vom täglichen Leben (S. 161-173),

- Bildorakel und konkrete Poesie im Abschnitt Bildgedichte (S. 93-100),

- gereimte Zweizeiler und Zungenbrecher im Abschnitt Tier-Zweizeiler (S. 103-110),

- Abzählreime, Spiel- und Necklieder im Abschnitt Anne, Pfanne, Badewanne, Apfelkuchen, du musst suchen! (S. 113-116),

- ABC-Spiele, Spiele mit Buchstaben und Ideogrammen im Abschnitt Aus dem Leben der Buchstaben (S. 119-124),

- makkaronische, auf Englisch-Deutsch aufgebaute Kinderreime im Abschnitt Rat und Cat (S. 127-132), 
- Rätsel im Abschnitt Rätselraten (S. 135-142),

- verdrehte Wörter und Spiele mit Buchstaben im Abschnitt Hier stimmt was nicht! (S. 145-152).

\section{Anregungen für den Unterricht}

Der nun folgende praktische Teil meines Artikels, der konkrete Anregungen für den Unterricht enthält, ist als Material für Studierende des Faches Deutsch als Fremdsprache für Kinder gedacht, das auf den Unterricht mit Kindern im Alter von etwa 6 bis 12 Jahren vorbereitet.

\subsection{Spiele mit Buchstaben}

Im Gedicht Vornamen- $A B C$ wird mit den Vornamen so gespielt, dass diverse Vornamen alphabetisch aneinandergereiht werden, wie z. B.:
Axel holt die Leiter,
Bärbel trägt sie weiter,
Christoph stellt sie auf,
Doris klettert auf.
(MAAR: 2007, 120)

Nachdem das Gedicht gelesen wurde und den Schülern der Aufbau des Gedichtes erklärt worden ist, erfinden die Schüler mit Hilfe ihrer eigenen bzw. wenig üblicher Vornamen ein ähnliches komisches Gedicht.

\subsection{Verdrehte Wörter}

Bei dieser Art des Kinderreims werden Buchstaben bzw. ganze Komponenten der Zusammensetzungen verdreht, sodass neue, unerwartete Zusammenhänge entstehen. Die Schüler lernen zunächst die Wörter aus dem Gedicht Verwandtschaft ordentlich lesen:

\section{Verwandtschaft}

Der Peter und die Silie gehören zur Familie.

Den Seller und den Rie sieht man dagegen nie. (MAAR: 2007, 146) 
Anschließend versuchen sie unter Verwendung von anderen, mindestens zweisilbigen Gemüse- bzw. Obstnamen (z. B. Kohlrabi, Mohrrübe, Birnenquitte, Schnittlauch oder Apfelsine) ein ähnliches Gedicht zu bilden.

Ähnlich wird auch beim nächsten Gedicht vorgegangen:

Land auf dem Sonntag

Im Scheinensonn

taubt eine Gurre

Im Schattenhaus

katzt eine Schnurre.

Es hummelt eine Brumm

Wie ein Wagenlast.

Sanft schweint ein Grunz

Vor der Wirtschaftsgast.

Im Weiherdorf

froscht tief der Tauch.

Oben am Dachhaus

schlotet der Rauch.

(MAAR: 2007, 148)

Nachdem die Wörter sinnvoll gelesen worden sind, fügen die Schüler ihre Stimmen zu weiteren Tieren hinzu, wie z. B.: „Der Hund bellt, das Pferd wiehert, das Schaf blökt" etc.

\subsection{Schüttelreim}

Die Schüler lernen zuerst das Prinzip des Schüttelreims als einer alten, seit dem 13. Jahrhundert existierenden Reimform, bei der die Anfangskonsonanten der letzten beiden betonten Silben miteinander vertauscht werden. Wegen der Anschaulichkeit sind die Anfangsbuchstaben der betreffenden Wörter schon in dem Gedichtband von Maar fett gedruckt.

Nicht sehr häufig rasen Hennen, öfter sieht man Hasen rennen.

(MAAR: 2007, 57)

Wenn Bienen statt zu brummen singen, muss man sie zum Summen bringen.

(MAAR: 2007, 58) 
Die Schüler erkennen, wie mit Buchstaben und Silben gespielt wird. Anschließend werden ähnliche Wortpaare gesucht, wie z. B.: Fischer schwimmen / Schwimmer fischen, Taucher fliegen / Flieger tauchen etc.

\title{
6.4 Abzählreim
}

Abzählreime werden beim Spielen angewendet. Sie unterstützen und entwickeln bei den Kindern das Gerechtigkeitsempfinden und tragen zur sozialen Integration bei. Das Kind erlebt das Gefühl, zufällig ausgewählt worden zu sein und dabei Freude zu empfinden bzw. nicht ausgewählt worden zu sein und trotzdem nicht unter der Zurücksetzung zu leiden. (vgl. FÜRST/HELBIG/SCHMITT: 2000).

Es ist wichtig, dass zuerst der Lehrer einen Abzählreim den Kindern auswendig spielerisch vorführt, wie z. B.:

\author{
Matschen, \\ knatschen, \\ wasserpatschen, \\ Klebe, \\ Knete, \\ Schlamm und Dreck. \\ Händewaschen - \\ DU bist weg! \\ (MAAR: 2007, 114)
}

\subsection{Zungenbrecher}

Mit dem Auswendiglernen von Zungenbrechern - wobei die Zungen- und Lippenmuskulatur trainiert wird - erreicht das Kind soziales Ansehen in der peergroup. Vom inhaltlichen Gesichtspunkt her macht vor allem das Aneinanderreihen von phonetisch ähnlichen Wörtern Spaß. Auch die bereits oben erwähnten Schüttelreime können als Zungenbrecher verwendet werden.

Im folgenden Text soll vor allem auf die schnelle fehlerlose Aussprache geachtet werden:

Sägen

Nur selten kommt der Sägehai

Zum Sägen in die Sägerei.

Den Sägefisch dagegen, den hört man öfter sägen.

(MAAR: 2007, 40) 


\subsection{Rätsel}

Rätsel gehören seit alten Zeiten zum volkstümlichen Dichtungsgut. Da die Menschen in ihrem Leben vor bösen und mythischen Mächten geschützt werden wollten, kleideten die unbekannten Autoren ihre Wünsche, Gedanken und Vorsätze in Rätsel. Die Kunst ein Rätsel zu erraten beweist Schlagfertigkeit, Weisheit und Bildung. Dem Kind verleiht diese Fähigkeit große Anerkennung in der Gruppe der Gleichaltrigen. Der Inhalt des Rätsels muss der Erfahrungswelt des Kindes entnommen sein.

Die einfachsten Rätsel erscheinen in der Form der Rätsel- bzw. Scherzfrage. Manchmal wird die Lösung bereits im Text verraten bzw. findet sich in einem Reimwort des Verses, wie z. B. in den folgenden beiden Gedichten:

Betrachte mal die LESELAMPE!

Steckt da nicht ein Vieh drin?

Ein graues Tier mit langen Ohren.

Schau doch mal genau hin!

(MAAR: 2007, 141)

Für ältere Kinder sind Rätsel geeignet, die höhere kognitive und assoziative Anforderungen stellen.

Ein mancher wär schon tot,

hätt es sein Auto nicht.

Doch bin ich auch ein Tier,

das hinterlistig sticht.

(MAAR: 2007, 140)

Im ersten Gedicht lautet die Lösung „Esel“, im zweiten „Bremse“.

\section{Schlusswort}

Kinderlyrik ist eine literarische Gattung, die innerhalb der Kinderliteratur scheinbar leicht abgegrenzt werden kann, die aber in sich eine komplexe Erscheinung darstellt. Sie wird oft als Privatlektüre beiseitegeschoben, kommt man jedoch mit ihr im Kindergarten bzw. in der Schule in Berührung, entdeckt man, welch großen Spielraum sie sprachlich, formal und intentional aufweist. Im Spiel mit der Sprache lernen die Kinder die Regeln der Sprache und gleichzeitig probieren sie aus, wie diese Regeln veränderbar und auf unkonventionelle Weise ersetzbar sind. Anhand der Gedichtsammlung Jaguar und Neinguar von Paul Maar habe ich mich mit den Sprachspielen dieses Autors beschäftigt. Zuerst habe ich diverse Formen 
der Kinderlyrik kategorisiert, so wie sie in dem Buch erscheinen. Als konkreter methodischer Beitrag für den Unterricht ist der letzte Abschnitt des Artikels gedacht, wo ich einige konkrete Formen von Sprachspielen methodisch-didaktisch behandelt habe. Bei den besprochenen Sprachspielen handelt es sich um Spiele mit Buchstaben, verdrehte Wörter, Schüttelreime, Abzählreime, Zungenbrecher und Rätsel.

\section{Primärquellen:}

MAAR (2007): Maar, Paul. Jaguar und Neinguar. Gedichte von Paul Maar. Hamburg: Friedrich Oetinger Verlag, 2007.

\section{Sekundärliteratur:}

BORN (1996): Born, Monika. Praktische Möglichkeiten der Hinführung zum Kinderbuch, in: Sahr, Michael/Born, Monika (Hg.): Kinderbücher im Unterricht der Grundschule. Baltmannsweiler: Schneider, 1996, 34-65.

FÜRST/HELBIG/SCHMITT (2000): Fürst, Andrea/Helbig, Elke/Schmitt, Vera. Kinderund Jugendliteratur. Neusäß: Kieser, 2000.

LANGE (1996): Lange, Günter. Phantastisches Erzählen im Werk Paul Maars, in: Franz, Kurt/Payrhuber, Franz-Josef (Hg.): Blickpunkt: Autor. (=Schriftenreihe der Deutschen Akademie für Kinder- und Jugendliteratur Volkach e. V.). Hohengehren: Schneider, 1996, 131-153.

PAYRHUBER (2000): Payrhuber, Franz-Josef. Kurzporträts von Paul Maar, Gudrun Mebs und Gunter Preuß, in: Franz, Kurt/Lange Günter/Payrhuber, Franz-Josef (Hg.): Kinderund Jugendliteratur zur Jahrtausendwende. Hohengehren: Schneider, 2000, 97-109. 